\title{
Vorwort der Herausgeber: Jacob Levy Moreno revisited - Ein schöpferisches Leben
}

\author{
Michael Wieser $\cdot$ Falko von Ameln
}

\section{Editorial: Jacob Levy Moreno revisited: a creative life}

Mit dieser Schrift möchten wir an Jakob Levy Morenos 125. Geburtstag vom 18. Mai 1889 und seinen 40. Todestag am 14. Mai 1974 erinnern. Es ist sozusagen ein Buch der Wandlung von Geburt zu Tod und Weiterleben des Schaffens.

Für den ersten Teil haben wir zu einer Art Geburtstagsrede eingeladen. Insbesondere diejenigen, die Leben und Werk von „doctor“ persönlich erlebt haben, sollen zu Wort kommen. An erster Stelle Ehefrau Zerka, die über mehr als 70 Jahre J.L.'s Leben und Werk mitgestaltet hat. Tochter Regina erzählt uns eine sehr persönliche Geschichte. Auch Neffe Joseph Jacob kommt nicht nur über die Begegnung mit seinem Onkel zum Psychodrama sondern führt die Verbindung fort als zweiten Vornamen. Die psychodramatische Tochter, Grete Leutz, ist vor mehr als 60 Jahren zur „Familie“ gestoßen und nicht nur mit der Übersetzung des Buches ,Who shall survive?" hat für sie die Anziehung begonnen. John Nolte ist derjenige, der Moreno am Ende des Lebens pflegerisch unterstützen konnte, nicht ohne zuvor Psychodrama gelernt zu haben. Dalmiro Bustos leitet neben seinen persönlichen Begegnungen mit Moreno schon über zu den theoretischen Teilen. René Marineau reflektiert über die Rolle des Biographen. Jonathan Moreno schließt den ersten Abschnitt mit Überlegungen zu seiner gerade fertiggestellten Biographie seines Vaters (Moreno 2014).

Der zweite Teil widmet sich historischenAnalysen zum Lebenswerk. Børge Kristoffersen untersucht den Begegnungsbegriff als expressionistischen Ausdruck und Sozialreform in Morenos Wiener Zeit. Friederike Scherr fokussiert auf die Anfänge der Soziometrie während des 1. Weltkriegs und beleuchtet das Archivmaterial. Krammer, Pfaffenwimmer, Scherr und Wieser legen die Vorarbeiten zu einem lebendigen Erinnerungsraum Moreno

\footnotetext{
Online publiziert: 23.05 .2014

(C) Springer Fachmedien Wiesbaden 2014

M. Wieser $(\bowtie) \cdot$ F. von Ameln

Institut für Psychologie, Alpen-Adria-Universität Klagenfurt, Universitätsstr. 65-67, 9020 Klagenfurt, Österreich

E-Mail: Michael.Wieser@aau.at

F. von Ameln

Am Diekschloot 11b, 26506 Norden, Deutschland
} 
Museum in Bad Vöslau dar. Tom Treadwell schließt den zweiten Abschnitt mit dem Blick auf Moreno als Pionier der Gruppenbewegung, die sich bis zu den Netzwerken der nun gebräuchlichen ,social media“ hinzieht.

Der dritte Teil bildet den inhaltlichen Kern des Buches. Unter der Überschrift „Moreno revisited“" geht es in sieben Beiträgen darum, Morenos Werk aus der heutigen Perspektive verschiedener Wissenschaftsdisziplinen einer kritischen Würdigung zu unterziehen und die Anschlussstellen an aktuelle Diskurse herauszuarbeiten. Morenos Ideen sind in vielerlei Hinsicht überraschend modern und werden in den heutigen Forschungsprogrammen oftmals weitergeführt, ohne dass dabei Morenos Beitrag angemessen gewürdigt würde.

Moreno bezog sich oft auf das Jahr 2000 und meinte, obwohl seine Ideen für das 20. Jahrhundert verfrüht seien, würde das nächste Jahrhundert ihm gehören [...]. Die Zukunft der Ideen Morenos wird durch die Wiederentdeckung von Moreno ,als Ganzem` gefördert werden, als eines Philosophen, der seine Philosophie in der konkreten Existenz jedes menschlichen Wesens verankerte. (Marineau 1989, S. 153)

Einerseits sollen die Beiträge in diesem Teil also die oftmals vergessenen Leistungen Morenos in Erinnerung rufen. Auf der anderen Seite soll der Blick auf Morenos Werk vor dem Hintergrund des aktuellen Standes in den Bezugswissenschaften auch Richtungen für die Weiterentwicklung der Psychodrama-Theorie aufzeigen. Denn, so Marineau (1989, S. 153), „die große Herausforderung der Zukunft liegt darin, [...] auf Morenos Fundamenten in kohärenter, systematischer und allumfassender Weise aufzubauen und sie umzugestalten“.

Es besteht dringender Bedarf, Morenos Ansichten zu integrieren, und sie in eine Sprache zu übersetzen, welche die Menschen des 21. Jahrhunderts sprechen. Verschiedene Disziplinen werden Wege finden müssen, um miteinander zu sprechen und zu interagieren. [...] Morenos umfassendes Konzept und die Aktionsmethoden können eine Hilfe sein, um eine gemeinsame Basis zu finden, und um neue Erkenntnisse für das Verständnis, sowohl des individuellen, ,privaten“ Lebens als auch für die Interaktionen mit anderen, zu entwickeln. (Marineau 2012, S. 39)

Dani Yaniv beleuchtet Morenos Werk vor dem Hintergrund neuerer Entwicklungen in der Neuropsychologie. Dabei zeigt Yaniv, wie aktuell Morenos Sozialtheorie angesichts der Erkenntnisse der Neuropsychologie erscheinen. Jürgen Kriz lädt zu einer Begegnung zwischen Morenos Gedanken und der personzentrierten Systemtheorie ein. Er lenkt den Blick auf das zentrale, aber häufig vernachlässigte Konzept der Szene. Michael Schacht gibt einen kenntnisreichen Überblick über aktuelle Entwicklungen in der Entwicklungspsychologie. So zeigen sich nicht nur die erstaunliche Aktualität von Morenos Gedanken, sondern auch Möglichkeiten für die Weiterentwicklung psychodramatischen Denkens und Arbeitens. Morenos soziometrische Arbeiten sind in der heutigen sozialen Netzwerkforschung aufgegangen. Jürgen Schunter und Martin Liebau zeigen diese Traditionslinie auf und erläutern, wie soziometrische Fragestellungen heute behandelt werden. Katharina Novy wirft aus soziologischer Perspektive einen Blick auf Morenos Forschungsansatz und seine Rollentheorie. Darüber hinaus beleuchtet sie, welchen Nutzen aktuelle soziologische Konzepte wie Habitus oder Diversity für die Psychodrama-Praxis entfalten können. Christoph Hutter führt die LeserInnen in seinem Beitrag durch Morenos reli- 
giöse Bilderwelt. Auf dieser Basis zeigt er, welche Aktualität Morenos Axiologie heute für Therapie, Beratung und Unternehmen hat. Hier knüpft der Beitrag von Falko von Ameln an, der aus Morenos Schriften eine implizite psychodramatische Organisationstheorie herausdestilliert und diese vor dem Hintergrund der aktuellen Organisations- und Führungsforschung diskutiert.

Für ein Verfahren, dessen Hauptanwendungsfeld nach wie vor die Psychotherapie darstellt, ist die klinische Psychologie zweifellos die wichtigste Bezugswissenschaft. Obwohl wir zahlreiche ExpertInnen im deutschsprachigen Raum, in den USA und anderen Ländern mit einem Call for Papers und direkt angesprochen haben, ist es uns nicht gelungen, jemanden zu finden, der bereit gewesen wäre, diesen wichtigen Beitrag zu übernehmen. Die einen haben ihre Identität aus dem akademischen Fach herausgelöst, die anderen haben sich zu wenig mit der Weiterentwicklung des Psychodramas beschäftigt. Das gilt teilweise auch für die Bereiche pädagogische und Sozial-Psychologie, aber zu diesen Zugängen hoffen wir auf weitere Publikationen.

Der abschließende vierte Teil würdigt Morenos Lebensleistung für die Arbeit in einigen wichtigen Anwendungskontexten. Reinhard Krüger beleuchtet Morenos Beitrag zur Psychotherapie mithilfe des Mentalisierungskonzeptes. In der szenischen Umsetzung von Mentalisierungsprozessen, wie sie das Psychodrama leistet, sieht er einen wichtigen Beitrag, den die Psychotherapie noch nicht ausreichend gewürdigt habe. Ferdinand Buers Beitrag erläutert, was das Psychodrama für Organisationsberatung, Supervision und Coaching leisten kann. Neben den Interpretationsfolien, die Morenos Werk für diese Formate bereithält, zeigt Buer auch ethische Implikationen für die psychodramatische Arbeit in Organisationen auf. Aus der Sicht von Thomas Schwinger wird das Psychodrama den Anforderungen der Sozialen Arbeit vor allem deshalb gerecht, weil seine szenische Arbeitsweise der dramatologischen Form des sozialen Lebens der KlientInnen entspricht. Schwingers Beitrag endet mit einem kritischen Blick auf die Grenzen der Anschlussfähigkeit und einem Plädoyer für die weitere Professionalisierung des Psychodramas: „Mehr Nüchternheit, weniger Begeisterung, mehr theoretische und methodische Weiterentwicklung.“ Ein geplanter Beitrag über Konvergenzen zwischen Psychodrama und Pädagogik musste leider kurz vor Drucklegung entfallen, was insbesondere deshalb bedauerlich ist, weil im Hinblick auf die theoretische Fundierung des Psychodramas in der Pädagogik noch großer Nachholbedarf besteht.

Wir bedanken uns bei allen AutorInnen sowie dem Verlag für das konstruktive Zusammenwirken und wünschen allen LeserInnen eine erkenntnisreiche Lektüre.

\section{Literatur}

Marineau, R. F. (1989). Jacob Levy Moreno, 1889-1974. Father of psychodrama, sociometry, and group psychotherapy. London: Tavistock.

Marineau, R. F. (2012). Die Integration von Morenos Erbe. In C. Stadler \& M. Wieser (Hrsg.), Psychodrama. Empirische Forschung und Wissenschaft (S. 35-44). Wiesbaden: Springer VS.

Moreno, J. D. (2014). Impromptu Man: JL Moreno and the Origins of Psychodrama, Encounter Culture, and the Social Network. New York: Bellevue Literary Press. 